\title{
Intraperitoneal cytology after laparoscopic radical hysterectomy with vaginal closure without the use of a manipulator for cervical cancer: a retrospective observational study
}

This article was published in the following Dove Press journal:

Cancer Management and Research

\author{
Tomohito Tanaka' \\ Shunsuke Miyamoto' \\ Shinichi Terada' \\ Yuhei Kogata' \\ Hiroshi Sasaki' \\ Satoshi Tsunetoh' \\ Takashi Yamada ${ }^{2}$ \\ Masahide Ohmichi' \\ 'Department of Obstetrics and \\ Gynecology, Osaka Medical College, \\ Takatsuki, Japan; ${ }^{2}$ Department of \\ Pathology, Osaka Medical College, \\ Takatsuki, Japan
}

\begin{abstract}
Purpose: Although laparoscopic radical hysterectomy (LRH) has been performed for patients with cervical cancer because of its minimal invasiveness, a recent large prospective study showed that LRH was associated with a lower rate of disease-free survival and overall survival. However, the reason for these results is not apparent. The aim of this study was to evaluate the tumor spillage during LRH with vaginal closure without the use of a manipulator.
\end{abstract}

Patients and methods: Twenty-four patients with cervical cancer underwent total LRH with vaginal closure. To evaluate the leakage of cancer cells during surgery, peritoneal cytology was performed before and after hysterectomy.

Results: Among 24 patients with cervical cancer, 2 had stage IA2 disease, 19 had stage IB1 disease and 3 had stage IIA1 disease. Two patients had lymph node metastasis. The median tumor size on final pathology was $9 \mathrm{~mm}$. No cancer cells were identified before or after hysterectomy in any patients.

Conclusion: Total LRH with vaginal closure did not increase the propensity for tumor spillage in the peritoneal cavity.

Keywords: cervical cancer, laparoscopy, radical hysterectomy, vaginal closure

\section{Introduction}

Laparoscopic surgery for patients with cervical cancer has been performed as a minimally invasive approach. A previous meta-analysis comparing laparoscopic radical hysterectomy $(\mathrm{LRH})$ with open radical hysterectomy $(\mathrm{ORH})$ for cervical cancer revealed that the oncologic outcomes did not differ between laparoscopic and open surgery. ${ }^{1,2}$ However, the Laparoscopic Approach to Cervical Cancer (LACC) trial, ${ }^{3}$ which is the first large randomized prospective study to compare laparoscopic and open approaches in radical hysterectomy, showed that the laparoscopic approach was associated with lower rates of disease-free and overall survival. A number of factors, including the period of treatment, tumor spillage due to the use of a manipulator and the effect of the insufflation gas on tumor-cell growth or spread, may explain the differences between the results of the prospective randomized study and the results of the previously reported retrospective study. However, the reason for this difference is unclear. In the current study, tumor cell spillage during LRH with vaginal closure, performed without the use of manipulator, was evaluated based on the intraperitoneal cytology.
Correspondence: Tomohito Tanaka Department of Obstetrics and Gynecology, Osaka Medical College, 2-7, Daigaku-machi, Takatsuki, Osaka 5698686, Japan

Tel $+8 \mid 72683$ |22।

$\mathrm{Fax}+81726841422$

Email gyn123@osaka-med.ac.jp 


\section{Materials and methods}

\section{Participants}

Between January 2016 and January 2019, a total of 24 Japanese cervical cancer patients underwent laparoscopic procedures at Osaka Medical College in Japan. All of the patients underwent total LRH with vaginal closure. The present study was approved by Osaka Medical College Clinical Research Review Board with the number of 2013-053 and was deemed to be in compliant with the Declaration of Helsinki. All patients provided their written informed consent.

\section{Cytopathologic procedure}

None of the patients had abnormal findings on preoperative endometrial cytology. The peritoneal wash cytology was collected on entering the peritoneal cavity, prior to surgical preparation and just after hysterectomy. The peritoneal cavity was washed with $20 \mathrm{~mL}$ of sterile physiological saline solution. The ascites was aspirated after peritoneal dispersion from the Douglas pouch and then sent for a cytological analysis. Thin-layer cell preparation was performed with a Shandon CytoSpin III Cytocentrifuge. Slides were stained with Papanicolaou and interpreted by experienced cytologists. The criteria for malignancy were adapted from Ziselman et al. ${ }^{4}$

\section{Surgical procedure}

Total LRH was performed as a standard 5-port technique without intrauterine manipulation in the Trendelenburg position. A 12-mm balloon trocar (Auto Suture Blunt Tip Trocar; Medtronic, Minneapolis, MN, USA), used for a $30^{\circ} 10-\mathrm{mm}$ laparoscope, was inserted under direct visualization (open laparoscopy) through an intraumbilical incision of $1.5 \mathrm{~cm}$. Three lateral $5 \mathrm{~mm}$ trocars were inserted (left and right lower abdominal quadrant, and left under the costal arch) for the ancillary instruments, and one $12-\mathrm{mm}$ trocar was placed midline suprapubically for further manipulations and the extraction of lymph nodes. The uterus was retracted using $5-\mathrm{mm}$ grasping forceps that were inserted into the left under the costal arch. Tubal ligation was performed using a hemoclip after the first cytology sampling on entering the peritoneal cavity. After pelvic lymph node dissection or sentinel node biopsy, LRH was performed with or without salpingo-oophorectomy. Briefly, the round and broad ligaments were coagulated and transected by a LigaSure (Medtronic, Minneapolis, MN, USA) or a bipolar coagulation device. After clamping the uterine artery lateral to the ureters and opening the ureteral tunnels, the ureters were unroofed and rolled laterally. The bilateral infundibulopelvic ligaments were coagulated and transected using an Enseal. The vesicouterine and uterosacral ligaments were also transected. After the parametrial nodes were dissected, the deep uterine vein was isolated and clamped. Then, the rectovaginal septum was separated. After the inferior hypogastric nerve was identified and divided laterally, the ligation of the bilateral paracolpium was performed using 1-0 polydioxanone suture. The corresponding paracolpos were then resected. Just before the vagina was entered, vaginal closure was performed transvaginally. Briefly, the cut line in a circle was determined transvaginally and 8-12 knots of 1-0 silk were placed (Figure 1). The vaginal mucosa was cut at $3 \mathrm{~mm}$ outside the knot (Figure 2). The vaginal cuff of the uterus side was closed by running sutures with 0 bicryl; the uterine cervix with cancer was covered with the vaginal wall to prevent to the spillage of tumor cells (Figure 3). The vagina was then entered on laparoscope cutting of the remaining vaginal tissue; a circumferential colpotomy was performed on the rim of the VAGI PIPE (Hakko Medical, Chikuma, Japan) with monopolar scissors. Just after removal of the uterus (Figure 4), with or without the adnexa, through the vagina using a Memo-bag, the second intraperitoneal wash cytology was collected. The vaginal cuff was then closed laparoscopically with running absorbable sutures.

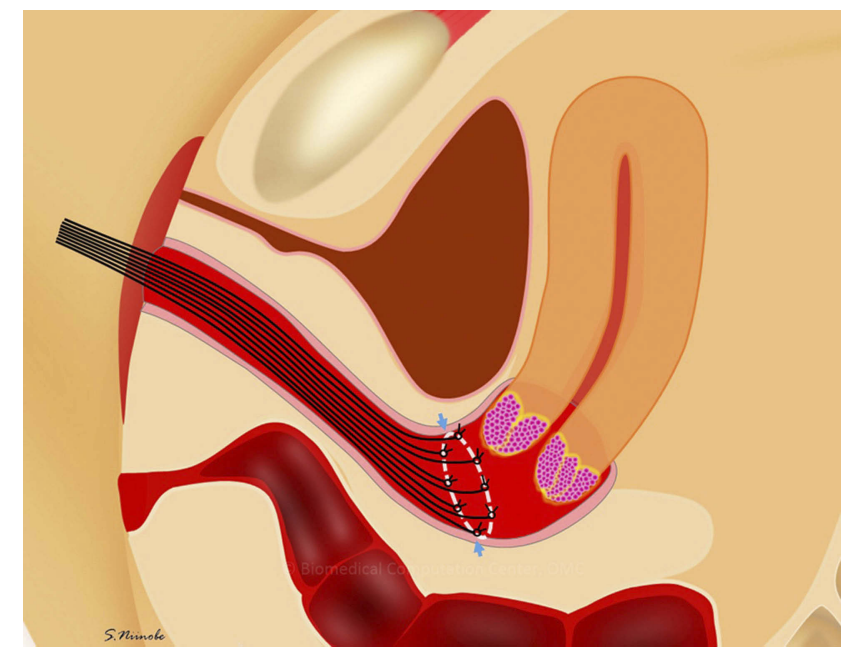

Figure I Vaginal closure at laparoscopic radical hysterectomy.

Note: The cut line (circle) was determined transvaginally and 8 to 12 knots of I-0 silk were placed (arrows). 


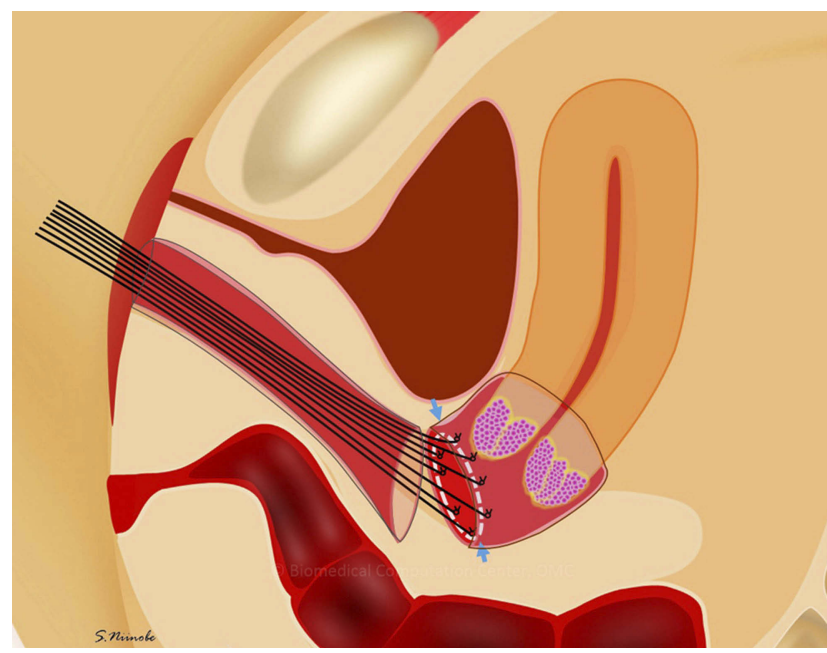

Figure 2 Vaginal closure at laparoscopic radical hysterectomy.

Note: Pulling the silk stretches the vaginal cut line. The vaginal mucosa was then cut at $3 \mathrm{~mm}$ outside the knot (arrows).

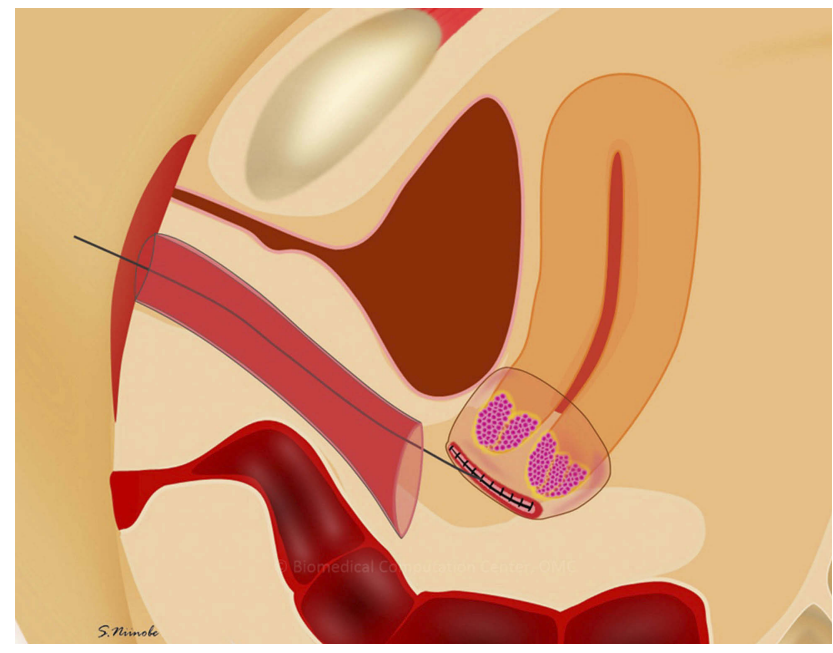

Figure 3 Vaginal closure at laparoscopic radical hysterectomy.

Note: The vaginal cuff on the uterus side was closed by running sutures with 0 bicryl; the uterine cervix with the tumor was covered with the vaginal wall to avoid spilling the tumor cells.

\section{Statistical analyses}

All of the statistical analyses were performed using the JMP software package (version 14.1.1). Continuous variables are expressed as the median and IQR or the mean $\pm \mathrm{SD}$.

\section{Results}

Table 1 shows the characteristics of 24 patients with cervical cancer who underwent LRH with vaginal closure. The mean age of the patients was $45.1 \pm 9.8$ years and the mean body mass index (BMI) was 21.2 \pm 3.4 . A total of 2 patients had International Federation of Gynecology and Obstetrics

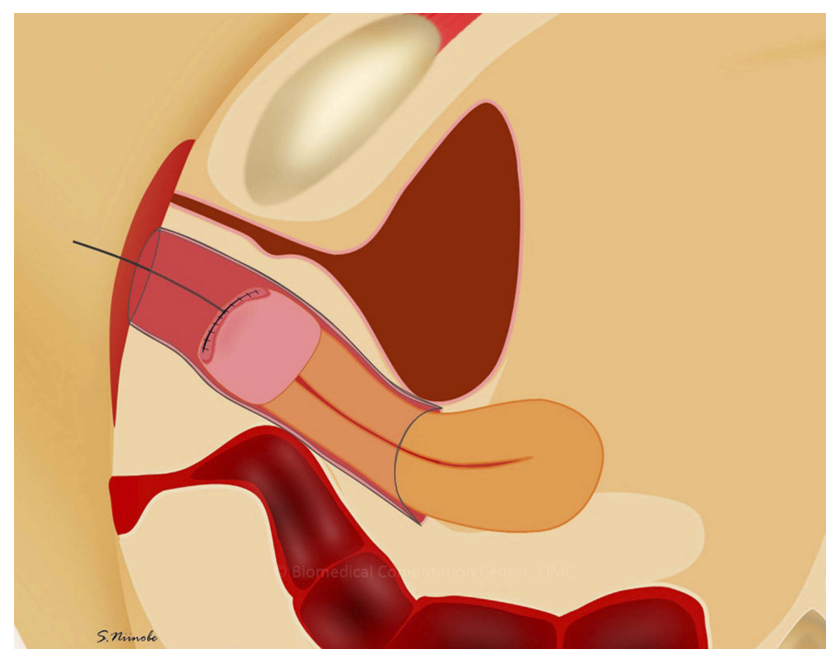

Figure 4 Vaginal closure at laparoscopic radical hysterectomy Note: After the vagina was entered on the laparoscopic cutting of the remaining vaginal tissue, the uterus was removed transvaginally.

(FIGO) stage IA2 disease, 19 had stage IB1 disease and 3 had stage IIA1 disease. Histologically, 12 patients had squamous cell carcinoma and 12 had adenocarcinoma. The median tumor size of the specimen was 9 (1-21) mm. Twentyone patients underwent performed sentinel node biopsy and 3 was performed systematic pelvic lymphadenectomy. Histologically, 2 patients had lymph node metastasis, 4 had lymphovascular involvement, 8 had deep stromal invasion and 1 had parametrial invasion. No patients had a bulky tumor or positive margin. Among the patients with these risk factors, 3 patients underwent concurrent chemoradiotherapy and 7 received chemotherapy as adjuvant therapy. No patient had positive peritoneal cytology before or just after hysterectomy. The median follow-up period was 12 (442) months. One patient developed recurrent disease at 2 months after surgery. Preoperatively, the patient had been diagnosed with stage IB disease that was $23 \mathrm{~mm}$ in diameter on magnetic resonance imaging. Fluorodeoxyglucose-positron emission tomography/computed tomography (FDGPET/CT) showed no signs of metastasis. LRH, bilateral salpingo-oophorectomy and pelvic lymph node dissection were performed with sentinel node mapping. The patient's peritoneal cytology was negative before and just after hysterectomy, after sentinel node biopsy and after pelvic lymph node dissection. The final diagnosis was cervical squamous cell carcinoma (diameter: $33 \mathrm{~mm}$ ). The patient had deep stromal invasion (more than half myometrial invasion), parametrial invasion and lymph node metastasis. One of 35 dissected lymph nodes, namely the left internal iliac node which was removed as sentinel lymph node, was positive for 
Table I The characteristics of the cervical cancer patients who underwent laparoscopic radical hysterectomy with vaginal closure

\begin{tabular}{|l|l|}
\hline Total number of patients & 24 \\
\hline Age* (years) & $45.1 \pm 9.8$ \\
\hline Body mass index & $21.2 \pm 3.4$ \\
\hline $\begin{array}{l}\text { FIGO stage } \\
\text { IA2 } \\
\text { IBI } \\
\text { IIAI }\end{array}$ & 2 \\
\hline $\begin{array}{l}\text { Histological type } \\
\text { Squamous cell carcinoma } \\
\text { Adenocarcinoma }\end{array}$ & 19 \\
\hline Tumor size on specimen ${ }^{\dagger}$ (mm) & 3 \\
\hline Lymphadenectomy & 12 \\
\hline $\begin{array}{l}\text { Sentinel } \\
\text { Systematic }\end{array}$ & 12 \\
\hline Lymph node metastasis & $9(1-21)$ \\
\hline Lymph vascular involvement & 21 \\
\hline Deep stromal invasion & 3 \\
\hline Bulky tumor & 2 \\
\hline Positive cut end & 4 \\
\hline $\begin{array}{l}\text { Parametrial invasion } \\
\text { Concurrent chemoradiotherapy }\end{array}$ & 8 \\
\hline Recurrence & 0 \\
\hline
\end{tabular}

Note: *Based on the ANOVA results (mean \pm SD). ${ }^{\dagger}$ Median (IQR).

Abbreviation: FOGO, International Federation of Gynecology and Obstetrics.

metastasis. The patient had no other risk factors (eg, positive cut end or lymphovascular involvement). After 2 courses of carboplatin plus paclitaxel as adjuvant chemotherapy, CT revealed recurrent masses on the vaginal stump and left iliac node. Bone metastasis was also found on the ischium. Concomitant chemoradiotherapy was scheduled; however, multiple lung and liver metastasis was found 1 month later. The patient died of the disease 6 months after LRH.

\section{Discussion}

In the current study, LRH with vaginal closure performed without the use of a manipulator did not increase the propensity for tumor spillage in the peritoneal cavity.
Over several decades, medical instruments have been developed and their use has grown. Laparoscopic surgery is one of the fields that has benefited most from these developments. Vaginal hysterectomy with laparoscopic adhesiolysis and the clamping and cutting of the infundibular ligament was first reported in the1980s. ${ }^{5,6}$ Reich et al reported the performance of laparoscopic hysterectomy in 1989. ${ }^{7}$ Their procedure included the clamping and cutting of the uterine artery and cardinal ligament. They also reported the performance of total laparoscopic hysterectomy-defined as the performance of all procedures (including vaginal closure) under laparoscopy-in 1992. ${ }^{8}$ These laparoscopic surgeries had been performed for patients with benign disease, including endometriosis, extensive fibroid disease, adnexal masses, adhesion from prior surgery or inflammatory disease. Laparoscopic surgery then came to be performed for malignant disease. Nezhat reported the performance of LRH for patients with cervical cancer ${ }^{9}$ in 1992. Since then, LRH for patients with cervical cancer has been performed in many institutions. Most published studies, including a meta-analysis comparing laparoscopic procedures with laparotomy for cervical cancer, showed that the oncologic outcomes did not differ between laparoscopic procedures and laparotomy. ${ }^{1,2,10,11}$ The largest retrospective matched cohort study was reported in 2012. The study revealed that LRH $(\mathrm{n}=263)$ was not associated with a higher risk of recurrence or death in comparison to ORH $(n=263)$. Furthermore, even in patients with tumors $>2 \mathrm{~cm}$ in diameter, LRH was not associated with an increased risk of recurrence or death. The 5-year recurrence-free survival rates of the LRH and ORH groups were $92.8 \%$ and $94.4 \%$, respectively $(p=0.5) .{ }^{10}$ They also reported on LRH for cervical cancer in patients with stage of IB2 and 2A2 disease. The 5-year disease-free survival rates of the LRH and ORH groups were $78 \%$ and $77 \%$, respectively $(p=0.78)$, and the 5 -year overall survival rate was $83 \%$ in both groups $(p=0.75) .{ }^{11}$ The LRH group showed a significantly lower volume of estimated blood loss and shorter postoperative hospital stay. Based on this, LRH is felt to be an oncologically safe alternative to ORH. Two metaanalyses reported that LRH was associated with better short-term outcomes in comparison to ORH and that the oncologic outcomes - including survival and the prognosis - did not differ between the groups. Wang et al reviewed 12 original studies to compare LRH $(n=754)$ to ORH $(\mathrm{n}=785)$ in 2015. In their study, there were no significant differences in 5 -year overall survival (HR $0.91, p=0.76$ ) or 
5 -year disease-free survival (HR 0.97, $p=0.91$ ). LRH was associated with a significant reduction of intraoperative blood loss, a reduced risk of postoperative complications and shorter hospital stay in comparison to ORH. ${ }^{1}$ Cao et al reviewed 22 studies, including 2922 cases ( $L R H, n=1230$; ORH, $n=1692$ ). The rates of disease-free survival, overall survival and recurrence did not differ among patients with balanced prognostic factors, including lymph node metastasis, stage $\geq$ IIB disease, nonsquamous histology, grade G3, lymphovascular space invasion, tumor size $>4 \mathrm{~cm}$ and positive parametrial and vaginal margin rates. LRH was associated with lower complication rates and a shorter time to the recovery of the bowel or bladder function in comparison to ORH. LRH was also associated with a longer operative time, less blood loss and a shorter length of hospital stay. They concluded that LRH is safe and that it is associated with a lower rate of operative complication than $\mathrm{ORH}^{2}{ }^{2}$ However, a large prospective study-the LACC trial-was published in 2018. The results had a great impact because LRH was associated with lower rates of disease-free survival and overall survival than ORH among women with early-stage cervical cancer, which is different from the findings of previous studies. In this trial, 319 patients were assigned to receive minimally invasive surgery (MIS) and 312 were assigned to receive ORH. In the MIS group, $84.4 \%$ of the patients underwent laparoscopy and $15.6 \%$ underwent robotassisted surgery. Most patients (91.9\%) had stage IB1 disease. MIS was associated with a lower rate of diseasefree survival than ORH (3-year rate, $91.2 \%$ vs $97.1 \%$; HR for disease recurrence or death from cervical cancer, 3.74; 95\% CI, 1.63-8.58) and a lower rate of overall survival (3year rate, $93.8 \%$ vs $99.0 \%$; HR for death from any cause, $6.00 ; 95 \%$ CI, 1.77-20.3). Although the reason for these differences was not known, the situation at recurrence may be a clue to solving the problem. Twenty-seven of the patients in the MIS group and 7 of the patients in the ORH group had recurrence. The number of patients with vaginal vault recurrence was 4 in the MIS group and 3 in the ORH group, but this did not differ to a statistically significant extent. However, all cases of nonvaginal vault pelvic recurrence occurred in the MIS group; thus, laparoscopic procedures may increase the risk of recurrence in the pelvic cavity. The use of a uterine manipulator might increase the propensity for tumor spillage. Furthermore, insufflation with $\mathrm{CO}_{2}$ gas may influence the growth or spread tumor cells. Our results proved that LRH with vaginal closure, performed without the use of a manipulator, was not associated with a propensity for tumor spillage.

The present study was associated with two major limitations that may reduce its value. First, the sample size was relatively small. Second, the study did not compare the outcomes of different surgical procedures, for example laparoscopy or laparotomy, the use or nonuse of a uterine manipulator, vaginal closure or nonvaginal closure. As such, our results must be confirmed in further studies.

In conclusion, LRH with vaginal closure without the use of a manipulator did not increase the propensity for tumor spillage. If laparoscopic surgery has an inferior prognostic outcome in comparison to open surgery, it is likely to be explained by other factors.

\section{Conclusion}

LRH with vaginal closure without the use of a manipulator did not increase the propensity for tumor spillage. If laparoscopic surgery has an inferior prognostic outcome in comparison to open surgery, it is likely to be explained by other factors.

\section{Acknowledgment}

Mr. Niinobe Shigefumi (Osaka Medical College Biomedical Computation Center) contributed to the artwork design.

\section{Author contributions}

All authors contributed to data analysis, drafting or revising the article, gave final approval of the version to be published, and agree to be accountable for all aspects of the work.

\section{Disclosure}

The authors report no conflicts of interest in this work.

\section{References}

1. Wang YZ, Deng L, Xu HC, Zhang Y, Liang ZQ. Laparoscopy versus laparotomy for the management of early stage cervical cancer. $B M C$ Cancer. 2015;15:928. doi:10.1186/s12885-015-1818-4

2. Cao T, Feng Y, Huang Q, Wan T, Liu J. Prognostic and safety roles in laparoscopic versus abdominal radical hysterectomy in cervical cancer: a meta-analysis. $J$ Laparoendoscopic Adv Surg Tech Part A. 2015;25(12):990-998. doi:10.1089/lap.2015.0390

3. Ramirez PT, Frumovitz M, Pareja R, et al. Minimally invasive versus abdominal radical hysterectomy for cervical cancer. $N$ Engl J Med. 2018;379(20):1895-1904. doi:10.1056/NEJMoa1806395

4. Ziselman EM, Harkavy SE, Hogan M, West W, Atkinson B. Peritoneal washing cytology. Uses and diagnostic criteria in gynecologic neoplasms. Acta Cytol. 1984;28(2):105-110.

5. Bruhat M, Mage G, Pouly J, Manhes H, Canis M, Wattiez M. Operative Laparoscopy. New York: McGraw Hill Inc.; 1992. 
6. Gomel V, Taylor P. Diagnostic and Operative Laparoscopy. St. Louis: Mosby-Year Book Inc.; 1995.

7. Reich H, DeCaprio J, McGlynn F. Laparoscopic hysterectomy. $J$ Gynecol Surg. 1989;5(213). doi:10.1089/gyn.1989.5.213

8. Reich H. Laparoscopic hysterectomy. Surg Laparosc Endosc. 1992;2 (1):85-88.

9. Nezhat CR, Burrell MO, Nezhat FR, Benigno BB, Welander CE. Laparoscopic radical hysterectomy with paraaortic and pelvic node dissection. Am J Obstet Gynecol. 1992;166(3):864-865. doi:10.1016/00029378(92)91351-a
10. Nam JH, Park JY, Kim DY, Kim JH, Kim YM, Kim YT. Laparoscopic versus open radical hysterectomy in early-stage cervical cancer: long-term survival outcomes in a matched cohort study. Ann Oncol. 2012;23(4):903-911. doi:10.1093/annonc/ $\operatorname{mdr} 360$

11. Park JY, Kim DY, Kim JH, Kim YM, Kim YT, Nam JH. Laparoscopic versus open radical hysterectomy in patients with stage IB2 and IIA2 cervical cancer. J Surg Oncol. 2013;108(1):6369. doi:10.1002/jso.23347

\section{Publish your work in this journal}

Cancer Management and Research is an international, peer-reviewed open access journal focusing on cancer research and the optimal use of preventative and integrated treatment interventions to achieve improved outcomes, enhanced survival and quality of life for the cancer patient.
The manuscript management system is completely online and includes a very quick and fair peer-review system, which is all easy to use. Visit http://www.dovepress.com/testimonials.php to read real quotes from published authors. 